\title{
Anomalies in the GRBs' distribution
}

\author{
Zsolt Bagoly $^{1}$, István Horváth ${ }^{2}$, Jon Hakkila ${ }^{3}$ and L. Viktor Tóth ${ }^{1}$ \\ ${ }^{1}$ Eötvös University, Budapest, email: zsolt.bagoly@ttk.elte.hu \\ ${ }^{2}$ National University of Public Service, Budapest \\ ${ }^{3}$ College of Charleston, Charleston, SC
}

\begin{abstract}
Gamma-ray bursts are the most luminous objects known: they outshine their host galaxies making them ideal candidates for probing large-scale structure. Earlier it has been found that the angular distribution of different groups of GRBs (long, intermediate and short) show deviation from the full randomness at different levels (e.g. Vavrek et al. (2008)).

In the current sample there are more than 361 GRBs with position and redshift: the large scale homogeneous and isotropic distribution were checked with nearest-neighbour tests and with the two-point correlation function.
\end{abstract}

Keywords. methods: data analysis, galaxies: high-redshift, gamma rays: bursts, cosmology: observations, (cosmology:) large-scale structure of universe

We have recently (Horvath et al. (2013), Horváth et al. (2014), Horváth et al. (2015)) identified a large clustering of gamma-ray bursts at $z \approx 2$ in the general direction of the constellations of Hercules and Corona Borealis. Angular test were applied on the $8 z$ /distance groups with the new data. Nearest-neighbour tests identify pairing consistent the previously found large, loose GRB cluster in the redshift range $1.6<z \leqslant 2.1$, with and estimated size to be about 2000-3000 Mpc across. We find the cluster to be centered at $l=88^{\circ}, b=63^{\circ}$ when it is modeled as having a disk-shaped symmetry. The $\chi^{2}$ probability that this clustering is random is $p=0.051$. This angular excess cannot be entirely attributed to known selection biases, making its existence due to chance unlikely. The scale on which the clustering occurs is disturbingly large: the underlying distribution of matter suggested by this cluster is big enough to question standard assumptions about universal homogeneity and isotropy (q.v. Balazs et al. (2015)).

In Bagoly et al. (2014) we reconstructed the empirical sky exposure function of a GRB-like angular point process using kernel based density estimators. Any selected group (e.g. Horváth et al. (2010), Veres et al. (2010)) can be tested for isotropy in this a way.

Using the empirical sky exposure function and the empirical radial distribution we determined the general spatial two-point correlation function. A strong peak was identified for separations of around $50 \mathrm{Mpc}$, with a probability of only $p=0.00996$ that this distribution is indicative of random fluctuations. This is caused by a pair of GRBs (GRB020819B and GRB050803), at a distance of $\approx 56 \mathrm{Mpc}$ from each other. The very low observered GRB to supercluster ratio makes the occurrence of this doublet interesting. One should mention that the method is quite robust as the neither the empirical sky exposure function nor the empirical radial distribution function is not expected to vary significantly on this scale. Further investigation is in progress.

Acknowledge

This research was supported by Hungarian OTKA grant NN111016 and by NASA EPSCoR grant NNX13AD28A.

\section{References}

Bagoly, Z., Balázs, L. G., Horváth, I., et al. 2014, in PoS(SWIFT10)060, Swift: 10 Years of Discovery, Proc. of Science, Rome, Italy, 2-5 December 2014

Balazs, L. G., Bagoly, Z., Hakkila, J. E., et al., 2015, MNRAS, 452, 2236-2246

Horváth, I., Bagoly, Z., Balázs, L. G., et al. 2010, ApJ, 713, 552

Horvath, I., Hakkila, J., \& Bagoly, Z. 2013, paper 33 in eConf Proceedings C1304143, 7th Huntsville GammaRay Burst Symposium, GRB 2013

Horváth, I., Hakkila, J., \& Bagoly, Z. 2014, A\&A, 561, L12

Horváth, I., Bagoly, Z., Hakkila, J., \& Tóth, L. V. 2015, A\&\&A, in print, http://arxiv.org/abs/1510.01933

Vavrek, R., Balázs, L. G., Mészáros, A., Horváth, I., \& Bagoly, Z. 2008, MNRAS, 391, 1741

Veres, P., Bagoly, Z., Horváth, I., Mészáros, A., \& Balázs, L. G. 2010, ApJ, 725, 1955 